\title{
Lower circulating platelet counts and antiplatelet therapy independently predict better outcomes in patients with head and neck squamous cell carcinoma
}

Saleh Rachidi ${ }^{1,2}$, Kristin Wallace ${ }^{2,3}$, Terry A Day ${ }^{2,4}$, Anthony J Alberg ${ }^{2,3}$ and Zihai Li $i^{1,2^{*}}$

\begin{abstract}
Background: Head and neck squamous cell carcinoma (HNSCC) mortality rates have not shown significant reduction in decades. Platelets are being implicated in having cancer-promoting roles, an observation supported by the adverse outcomes associated with thrombocytosis in many malignancies associated with thrombocytosis. However, the prognostic significance of platelet counts in HNSCC is unknown. Here, we comprehensively investigate the predictive value of platelet counts at diagnosis and post-diagnosis antiplatelet treatment in the overall survival of HNSCC patients.

Methods: The study population consists of 1051 pathologically confirmed HNSCC cases diagnosed between years 2000 and 2012 in a tertiary medical center. Platelet count was investigated as a predictor of survival by fitting Cox Proportional Hazards (CPH) regression models to generate Hazard Ratios (HR) and 95\% confidence intervals $(\mathrm{Cl})$, while adjusting for age, sex, race, stage, treatment and smoking status. Finally, we evaluated the association between overall survival and antiplatelet medication intake after diagnosis.

Results: Multivariable analysis showed an increased death rate in patients with thromobocytosis $[\mathrm{HR} 2.37,95 \% \mathrm{Cl}$ 1.60-3.50)] and high normal platelet counts [HR 2.20,95\% Cl 1.58-3.05] compared to the reference middle normal group. Post-diagnosis treatment with antiplatelet medications was inversely associated with death rate [HR 0.76 , 95\% Cl 0.58-0.99].

Conclusions: Higher platelet counts were associated with poorer prognosis in HNSCC patients, whereas antiplatelet agents were associated with better prognosis. Antiplatelet agents warrant evaluation in preclinical and clinical settings as a way to improve survival in HNSCC.
\end{abstract}

\section{Introduction}

Head and neck cancer is common in many areas across the world and is mainly linked to tobacco smoking, alcohol consumption and Human Papilloma Virus (HPV) infections. Fifty-five thousand cases are newly diagnosed each year in the United States [1] and 550,000 worldwide [2]. Recent work has shown hematological parameters including neutrophils, lymphocytes and monocytes to predict outcome in head and neck cancers [3-5]. In

\footnotetext{
* Correspondence: zihai@musc.edu

'Department of Microbiology and Immunology, 86 Jonathan Lucas, Suite 612, Charleston, SC 29425, USA

${ }^{2}$ Hollings Cancer Center, 86 Jonathan Lucas, Suite 612, Charleston, SC 29425, USA

Full list of author information is available at the end of the article
}

addition, pretreatment thrombocytosis correlated with shorter survival in oral squamous cell carcinoma [6]. Intriguingly, thrombocytosis, which is highly prevalent in cancer patients [7], is a poor prognostic indicator in a number of cancers [8-13].

The relation between platelets and cancer progression suggests a possible role that extends beyond their hemostatic function [14,15]. More recently, platelets have been recognized as mediators of other regulatory functions in physiology such as angiogenesis, wound healing and immunomodulation [15]. Platelets secrete cytokines and growth factors such as TGF- $\beta$ [16], VEGF [17], MMP-2, PF4 [18] and PDGF [19], which in turn induce hallmarks of cancer progression such as epithelial-mesenchymal transition (EMT) [20], angiogenesis, cell migration and/or 
proliferation. For example, PDGF induces the dimerization of PDGFR and EGFR, resulting in EGFR transactivation [21]. Platelet-derived TGF- $\beta$ also acts on cancer cells to activate the Smad and NF- $\mathrm{kB}$ pathways, thus promoting cancer metastasis [22]. As a perpetuating mechanism, cancer cells can also produce soluble mediators such as IL-6 and GM-CSF, which stimulate thrombopoiesis [23,24]. Interestingly, the use of drugs with antiplatelet activity, such as aspirin, is associated with lower incidence and better prognosis in colon cancer and other cancers $[25,26]$.

In the present investigation, we examine closely and systemically the relationship between platelet count in the peripheral venous blood and survival in head and neck squamous cell cancer (HNSCC), including oral, pharyngeal and laryngeal cancers. In particular, we investigate the prognostic significance of platelet counts within the normal and abnormal ranges to determine if there is a linear association between platelet number and clinical outcome. To further investigate the role of platelets in HNSCC, we studied the association of medications known to interfere with platelet function; anti-platelet medications and nonsteroidal anti-inflammatory drugs (NSAIDs) [27], with survival of HNSCC patients.

\section{Subjects and methods}

\section{Patient inclusion and exclusion criteria}

The Cancer Registry at Hollings Cancer Center, Medical University of South Carolina (MUSC) was used to identify all cases of HNSCC (squamous cell cancers of the oral cavity, pharynx and larynx). The study population was comprised of histologically confirmed cases diagnosed between January 1, 2000 and June 30, 2012. After excluding patients diagnosed with a second primary cancer between January 1, 1993 (the earliest date recorded) and June 30, 2013, there were 1376 HNSCC patients available for the study. Patients without recorded platelet counts were also excluded, resulting in a total of 1051 patients available for analysis. The follow-up period ranged between 2 weeks and 156 months, with a median 25.7 months.

\section{Data collection}

The MUSC Institutional Review Board approved all study activities. For each case, we abstracted data on demographic characteristics, clinical and pathological variables at diagnosis, treatment received and outcome using two different data sources: the Hollings Cancer Center (HCC) cancer registry and the MUSC Clinical Data Warehouse (CDW). The registry is part of a state mandated data system that collects cancer incidence on all cases in South Carolina. The CDW is a single, secure and integrated database extracted from the MUSC OACIS Clinical Data Repository, which includes patient demographics, ICDcoded diagnoses, ICD-coded procedures, medications and laboratory test results. These databases were subsequently linked, in a blinded fashion, through an honest broker at the CDW, and entered into a secured study database.

Independent variables obtained from the CDW included socio-demographic characteristics (age at diagnosis, sex and race); pre-treatment platelet count, lifestyle factors including smoking status (never, former and current) and alcohol use (never, former and current); and use of plateletinterfering medications, namely NSAIDs or antiplatelet medications, after diagnosis. NSAIDs included aspirin, celecoxib, rofecoxib, valdecoxib, diclofenac, etodolac, ibuprofen, indomethacin, ketorolac, meloxicam, nabumetone, naproxen, oxaprozin, sulindac and choline magnesium trisalicylate. Antiplatelet medications included cilostazol, clopidogrel, prasugrel, ticlopidine, abciximab, eptifibatide, tirofiban, anagrelide, dipyridamole, dipyridamole and aspirin. Intake of medications was based on the inpatient records of the CDW. Tumor-related variables obtained from the registry included tumor grade (well differentiated, moderately differentiated, poorly differentiated/ undifferentiated); location of the primary tumor (oral cavity, pharynx, larynx); TNM stage (I, II, III, IV); and receipt of all first-line therapies (chemotherapy, surgery, radiation and/or other). Because there were only 5 cases with undifferentiated grade, we combined these with poorly differentiated grade into a single category for the purposes of analysis.

\section{Data analysis}

The main study outcome was overall survival. Survival time was calculated as the time from diagnosis with HNSCC to death from any cause through July 19, 2013. Subjects alive as of this date were censored at the end of follow-up. Kaplan-Meier methods were used to generate median survival time and corresponding 95\% confidence intervals for five classes of platelet count (low, low-normal, mid-normal, high-normal and high). Abnormal platelet count was defined as thrombocytopenia ("low", <150,000/ $\mu \mathrm{L}$ ) or thrombocytosis ("high", $\geq 400,000 / \mu \mathrm{L}$ ). Within the normal range (150-399), we a priori sub-divided patients into approximate thirds [i.e. low-normal (150-229), mid-normal (230-314) and high-normal (315-399)]. Kaplan-Meier curves were plotted to graphically assess the relationship between the five categories of platelets (described above) and survival. The log-rank test was used to assess statistical significance.

We evaluated the association between the 5 platelet categories and survival by fitting univariate Cox Proportional Hazards $(\mathrm{CPH})$ regression models. We also analyzed platelets as a continuous variable within the normal range. We chose to restrict the analysis to the normal range because the relationship between platelets and death was nonlinear (u-shaped curve) in the lower tail of the distribution. To control for potential confounding variables, we performed univariate $\mathrm{CPH}$ for all other variables in Table 1 
Table 1 Demographic and clinical characteristics within the five categories of platelet counts

\begin{tabular}{|c|c|c|c|c|c|c|}
\hline \multirow[b]{2}{*}{ Variable } & \multicolumn{6}{|c|}{ Platelet count categories } \\
\hline & $\begin{array}{l}\text { Low (1-149) } \\
\mathrm{N}=67\end{array}$ & $\begin{array}{l}\text { Low normal } \\
(150-229) \mathrm{N}=361\end{array}$ & $\begin{array}{l}\text { Mid normal } \\
(230-314) \mathrm{N}=379\end{array}$ & $\begin{array}{l}\text { High normal } \\
(315-399) \mathrm{N}=169\end{array}$ & $\begin{array}{l}\text { High }(\geq 400) \\
\mathrm{N}=75\end{array}$ & $p$-value \\
\hline Age-yrs. Mean (SD) & $60.0(10.7)$ & $61.0(10.8)$ & $58.4(11.9)$ & $58.0(10.8)$ & $58.2(11.5)$ & 0.009 \\
\hline Male-no. (\%) & $55(82)$ & $302(84)$ & $273(72)$ & $117(69)$ & $53(71)$ & $<0.0001$ \\
\hline Race-no. (\%) & & & & & & $<0.0001$ \\
\hline Caucasian & $48(72)$ & $287(79)$ & $293(77)$ & $116(69)$ & $42(56)$ & \\
\hline African American & $18(27)$ & $67(19)$ & $83(22)$ & $52(31)$ & $33(44)$ & \\
\hline Other & $1(1.5)$ & $7(2)$ & $3(1)$ & $1(0.5)$ & $0(0)$ & \\
\hline Smoker-no. (\%) & & & & & & $<0.0001$ \\
\hline Never & $6(9)$ & $63(19)$ & $67(19)$ & $18(11)$ & $4(6)$ & \\
\hline Former & $20(32)$ & $125(38)$ & $111(32)$ & $40(25)$ & $14(20)$ & \\
\hline Current & $37(59)$ & $145(43)$ & $173(50)$ & $102(64)$ & $52(74)$ & \\
\hline Alcohol - no. (\%) & & & & & & 0.02 \\
\hline Never & $17(27)$ & $99(30)$ & $108(29)$ & $36(23)$ & $7(10)$ & \\
\hline Former & $13(20)$ & $55(17)$ & $54(14)$ & $26(17)$ & $9(14)$ & \\
\hline Current & $34(53)$ & $174(53)$ & $177(46)$ & $94(60)$ & $50(76)$ & \\
\hline Stage & & & & & & 0.034 \\
\hline । & $6(11)$ & $42(15)$ & $42(14)$ & $8(7)$ & $5(8)$ & \\
\hline$\|$ & $9(17)$ & $37(13)$ & $35(12)$ & $10(8)$ & $4(6)$ & \\
\hline III & $9(17)$ & $60(21)$ & $50(17)$ & $18(15)$ & $9(14)$ & \\
\hline IV & $29(55)$ & $147(51)$ & $168(57)$ & $87(71)$ & $47(72)$ & \\
\hline Grade & & & & & & 0.90 \\
\hline Undetermined & $20(30)$ & $106(29)$ & $103(27)$ & $42(25)$ & $16(21)$ & \\
\hline I. Well Differentiated & $9(13)$ & $43(12)$ & $49(13)$ & $18(11)$ & $8(11)$ & \\
\hline II. Moderately & $29(43)$ & $152(42)$ & $164(43)$ & $80(47)$ & $41(55)$ & \\
\hline III. Poorly or undifferentiated & $9(13)$ & $59(17)$ & $63(17)$ & $29(17)$ & $10(13)$ & \\
\hline Primary Site & & & & & & 0.09 \\
\hline Oral & $34(51)$ & $171(47)$ & $190(50)$ & $100(59)$ & $34(45)$ & \\
\hline Pharynx & $13(19)$ & $101(28)$ & $107(28)$ & $42(25)$ & $18(24)$ & \\
\hline Larynx & $20(30)$ & $89(25)$ & $82(22)$ & $27(16)$ & $23(31)$ & \\
\hline Post-diagnosis NSAIDs and/or Anti-platelets & & & & & & 0.33 \\
\hline No no. (\%) & $48(72)$ & $259(72)$ & $277(73)$ & $133(79)$ & $60(80)$ & \\
\hline Yes no. (\%) & $19(28)$ & $102(28)$ & $102(27)$ & $36(21)$ & $15(20)$ & \\
\hline
\end{tabular}

All variables had complete data $(n=1051)$ except: smoking status $(n=977)$, alcohol use $(n=953)$, clinical stage $(n=822)$ and tumor grade, where the undetermined category accounted for $n=247$ cases.

(i.e., age, sex, race, smoking status, alcohol use, treatment, tumor grade, location and clinical stage). Multivariable $\mathrm{CPH}$ models were then fit for the platelet variable and all other variables that were found to have a p-value of $<0.20$ in the univariate analysis. Variables were retained in the final CPR model if they were significant at $\mathrm{p}<0.05$. Additionally, for each model, we examined two-way interactions between platelet measures and variables that were significant in the multivariable model. Interactions were retained in the final model if they were significant at $\mathrm{p}<0.05$.
The methodology described above was used to examine the relationship between post-diagnosis use of NSAIDs/ antiplatelets and survival. The association between any post-diagnosis NSAIDs/antiplatelet medication use (Yes or No) and survival was assessed while adjusting for age, race, smoking, treatment, tumor site, tumor grade, platelet level (continuous, or platelet classes) and clinical stage. We also examined the association of medication use and survival while excluding those with pre-diagnostic thrombocytopenia (who would not be expected to benefit from antiplatelet medications). The interaction between 
Table 2 Relative hazard of death in HNSCC patients based on platelet counts

\begin{tabular}{|c|c|c|c|c|c|}
\hline Variables & Low $(<150) N=67$ & $\begin{array}{l}\text { Low normal } \\
(150-229) \mathrm{N}=361\end{array}$ & $\begin{array}{l}\text { Mid-normal } \\
(230-314) \mathrm{N}=379\end{array}$ & $\begin{array}{l}\text { High normal } \\
(315-399) \mathrm{N}=169\end{array}$ & HIGH $(\geq 400) \mathrm{N}=75$ \\
\hline Number dead (\%) & $37(55)$ & $150(42)$ & $146(39)$ & $105(62)$ & $53(71)$ \\
\hline Median Survival Months $(95 \% \mathrm{Cl})$ & $37.7(20.7-84.8)$ & $64.0(53.6-78.9)$ & $74.7(60.7$ 100.4) & $23.8(17.7-36.7)$ & $16.3(11.8-29.2)$ \\
\hline $\mathrm{p}$-value for difference & & & & & $<0.0001$ \\
\hline \multicolumn{6}{|l|}{ Overall Survival } \\
\hline Unadjusted HR (95\% CI) & $1.52(1.06-2.18)$ & $1.07(0.85-1.34)$ & 1.0 (reference) & $1.98(1.54-2.55)$ & $2.64(1.92-3.61)$ \\
\hline Adjusted HR (95\% Cl) & $1.50(0.96-2.35)$ & $1.25(0.93-1.68)$ & 1.0 (reference) & $2.20(1.58-3.05)$ & $2.37(1.60-3.50)$ \\
\hline \multicolumn{6}{|l|}{ HR $(95 \% \mathrm{Cl})$ by Stage } \\
\hline I $(n=103)$ & $0.41(0.05-.3 .31)$ & $1.18(0.33-4.20)$ & 1.0 (reference) & $1.60(0.27-9.54)$ & $0.35(0.03-4.18)$ \\
\hline$\|(n=95)$ & $1.99(0.52-7.67)$ & $1.40(0.57-3.38)$ & 1.0 (reference) & $1.54(0.48-4.99)$ & $1.83(0.45-7.40)$ \\
\hline III $(n=146)$ & $0.74(0.16-3.40)$ & $1.32(0.62-2.82)$ & 1.0 (reference) & $2.43(0.91-6.50)$ & $1.86(0.53-6.45)$ \\
\hline IV $(n=478)$ & $2.33(1.34-4.10)$ & $1.18(0.78-1.78)$ & 1.0 (reference) & $2.50(1.66-3.76)$ & $2.47(1.55-2.93)$ \\
\hline
\end{tabular}

Adjusted model controls for age, race, site, grade, treatment, smoking and stage.

$P$ for interaction between stage and classes of platelets $p=0.31$.

post-diagnostic NSAIDs/antiplatelet use and platelet classification survival was also evaluated.

\section{Results}

Relationship between platelet counts and clinical characteristics in HNSCC

Age inversely correlated with platelet counts, where patients in the low normal and low categories were slightly older than those in the other groups (Table 1). The majority of patients were males, but females were enriched in the higher platelet groups (mid-normal, high normal and high) (Table 1). In addition, African American patients were more represented in the thrombocytosis group where they comprised more than half of that population. Current smoking and alcohol consumption in turn were significantly associated with higher platelet counts (Table 1). Intriguingly, compared to those with stage I-III disease, higher platelet counts were observed in stage IV HNSCC (Table 1), although platelet levels did not significantly correlate with tumor grade or anatomic site (Table 1). No significant association was observed between intake of antiplatelet medications/NSAIDs and the platelet count. Finally, the proportion of deceased patients was higher in the high normal and thrombocytosis groups.

\section{Higher platelet counts are associated with worse overall survival}

Compared to those in the mid-normal category, patients in the high normal (adjusted HR 2.20; 95\% CI 1.58-3.05) and thrombocytosis (adjusted HR 2.37; 95\% CI 1.60-3.50) groups had more than two times higher death rates (Table 2 and Figure 1). The results for patients with platelet counts below the mid-normal category also trended toward poorer survival with adjusted hazard ratios of 1.25 (95\% CI $0.93-1.68)$ in the low normal and 1.50 (95\% CI $0.96-2.35)$ in the low categories, although these differences were not statistically significant.

Strikingly, when platelet count was modeled as a continuous variable within the normal range, an increase of platelets by $100,000 / \mu \mathrm{L}$ resulted in a $32 \%$ increase in the hazard ratio $(\mathrm{p}=0.001)$ in the unadjusted model. The increase in hazard was $30 \%(\mathrm{p}=0.01)$ after controlling for age, race, treatment, tumor site, tumor grade, smoking and stage.

The univariate association between platelet count and risk of death was assessed for three tumor sites (oral, pharynx, and larynx). Patients with thrombocytosis compared to those in the mid-normal range had a HR of 4.35 (95\% CI 2.75-6.87) for oral cancer, 2.41 (95\% CI 1.32-4.44) for pharyngeal cancer, and 1.48 (95\% 0.78-2.80) for laryngeal cancer.

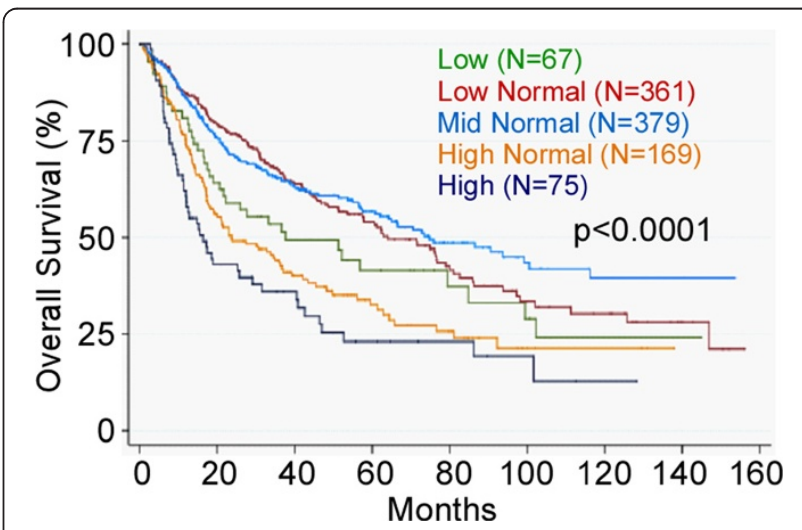

Figure 1 High normal platelets and thrombocytosis are associated with worse overall survival. (log-rank $p<0.0001)$. 
Table 3 Association of antiplatelet medications and risk of death

\begin{tabular}{llll}
\hline & \multicolumn{2}{l}{ HR 95\% Cl } & p-value \\
\hline Antiplatelet & No & Yes & \\
Unadjusted & 1.0 & $0.73(0.58-0.92)$ & 0.0008 \\
Adjusted* & 1.0 & $0.76(0.58-0.99)$ & 0.04
\end{tabular}

Antiplatelet medications in $\mathbf{5}$ categories of platelet counts

\begin{tabular}{llll} 
Low & 1.0 & $1.74(0.74-4.09)$ & 0.20 \\
Low Normal & 1.0 & $0.88(0.55-1.42)$ & 0.58 \\
Mid Normal & 1.0 & $0.68(0.42-1.11)$ & 0.12 \\
High normal & 1.0 & $0.87(0.48-1.51)$ & 0.64 \\
High & 1.0 & $0.42(0.17-1.05)$ & 0.06 \\
\hline
\end{tabular}

*Adjusted for age, race, tumor site, grade, treatment, platelet count and smoking.

\section{Antiplatelet medications are associated with longer overall survival}

Patients who were prescribed antiplatelet medications or NSAIDs after the date of diagnosis had a reduced risk of death compared to the other patients [HR 0.76, 95\% CI 0.58- 0.99] after controlling for age, race, stage, grade, tumor site, treatment, smoking status, and platelet count (continuous) (Table 3 and Figure 2). The HR for medication use and death rate was 0.80 (95\% CI 0.61-1.05) when we adjusted for age, race, stage, grade, tumor site, treatment, smoking status, and platelet classification. The association between the use of antiplatelet medications and death was stronger, however, when patients with thrombocytopenia were excluded: HR 0.73 (95 CI 0.55-0.98; $\mathrm{p}=0.04$ ). The association between medication intake and survival was assessed within each of the five platelet categories. Within the normal platelet range, intake of NSAIDs/antiplatelet conferred variable HRs in the protective direction, none of which was statistically significant. However, the protective association with intake of antiplatelet medications was strongest in the thrombocytosis group compared to those not taking medications [HR 0.42, 95\% CI 0.17-1.05), $\mathrm{p}=0.06$ ] (Table 3).

\section{Discussion}

The present study observed a clear association between higher platelet counts and worse overall survival in HNSCC. The results suggested that higher platelet levels, even in the normal range, are associated with poorer survival.

The evidence that the observed association between platelets and overall survival is genuine was solidified by the observation that the post-diagnostic intake of medications known to interfere with platelet function was associated with reduced risk of death in HNSCC patients, particularly among those with thrombocytosis. The fact that pharmacologic interventions aimed at lowering platelets were associated with prolonged survival provides quasi-experimental evidence that platelets are indeed linked with poor prognosis, reducing the likelihood that the observed association was due to bias or confounding factors. However, this evidence would be even stronger if it was clear that the observed association was due to an anti-platelet effect of these medications, but at the present time it cannot be ruled out that this association is attributable to the anti-inflammatory effects of many anti-platelet medications. However, given the recognized role of platelets in promoting cancer metastasis in preclinical animal models [22], it is likely that antiplatelet agents confer a beneficial effect and improve overall survival in cancer patients.

A limitation of our study is that we were unable to account for the amount and duration of intake of these agents, nor the start of their intake after diagnosis. The measurement error introduced likely resulted in bias toward the null hypothesis, but future studies with more detailed measurement of the anti-platelet medications are needed to refine our understanding on this issue.

Of note, our results suggested that low platelet counts $(<150,000 / \mu \mathrm{L})$ were associated with a trend towards worse overall survival compared to those in the low normal and mid-normal groups. However, the difference between the two groups did not reach statistical significance and is thus inconclusive. Alternatively, even if the null
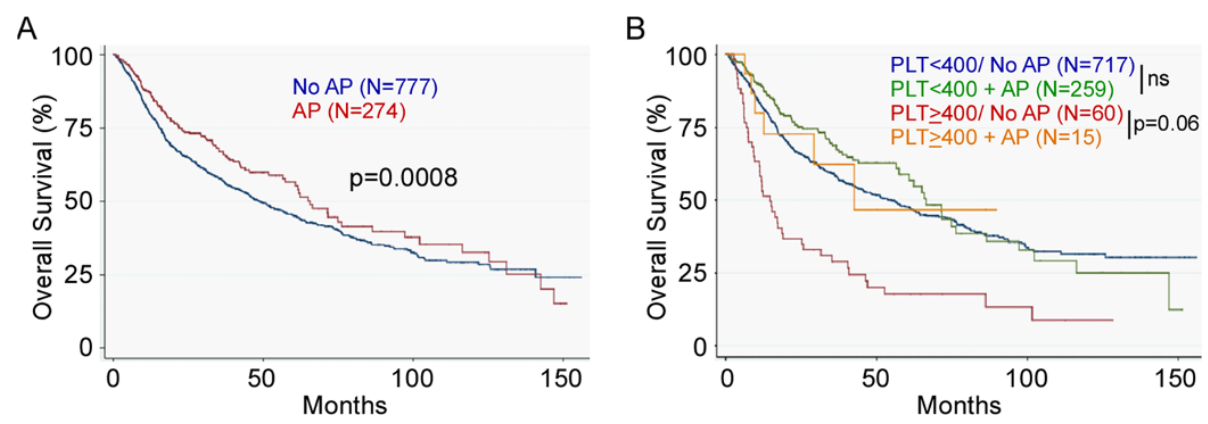

Figure 2 Antiplatelets/NSAIDs are inversely associated with survival in HNSCC. A. Intake of these medications confers better outcomes in the general HNSCC patient population, $\mathbf{B}$. The benefit associated with intake of antiplatelets/NSAIDs is particularly observed in the thrombocytosis group, $\mathrm{HR}=0.42, \mathrm{p}=0.06$. AP: Antiplatelets. ns: Not significant. 
hypothesis is rejected by future studies with increased sample size, the possible explanations include pathological thrombocytopenia acting as a marker of poor hematological function associated with advanced disease. This consideration emphasizes the importance of considering a potential U-shaped relationship between platelet counts and cancer survival in future research on this topic.

\section{Conclusions}

In conclusion, higher platelet counts were strongly associated with worse overall survival in a large cohort of head and neck cancer patients, whereas anti-platelet medications were associated with better survival. These findings, if replicated, have direct translational implications. Given the worse prognosis associated with higher platelet counts, and the potentially protective effect of platelet inhibitors in counteracting the effect of platelets, the investigation of anti-platelet agents in the treatment of HNSCC is warranted, especially that such agents are widely available and approved for other indications.

\section{Competing interests}

The authors declare that they have no competing interests.

\section{Authors' contributions}

SR and KW performed the research, analyzed and interpreted the data. TAD and AJA contributed to the discussion and analysis of some of the data. All authors read and approved the final manuscript. SR and ZL conceived the idea. ZL supervised the study.

\section{Acknowledgements}

This study was supported in part by National Institutes of Health grants Al070603, Al077283 (to Z.L.), P30 CA138313 (to the Hollings Cancer Center, Medical University of South Carolina). We are indebted to Drs. Jennifer Wu, Stephen Tomlinson, Harry Drabkin, and members of our laboratories for helpful discussions during the course of this study. Z.L. is Sally Abney Rose endowed chair in stem cell biology and therapy.

\section{Author details}

'Department of Microbiology and Immunology, 86 Jonathan Lucas, Suite 612, Charleston, SC 29425, USA. ${ }^{2}$ Hollings Cancer Center, 86 Jonathan Lucas, Suite 612, Charleston, SC 29425, USA. ${ }^{3}$ Department of Public Health Sciences, 68 President Street, BE 103, Charleston, SC 29425, USA. ${ }^{4}$ Head and Neck Tumor Center, Department of Otolaryngology-Head and Neck Surgery, Medical University of South Carolina, 171 Ashley Avenue, Charleston, SC 29425, USA.

Received: 28 May 2014 Accepted: 6 August 2014

Published online: 27 September 2014

\section{References}

1. Siegel R, Ma J, Zou Z, Jemal A: Cancer statistics, 2014. CA Cancer J Clin 2014, 64:9-29.

2. Jemal A, Bray F, Center MM, Ferlay J, Ward E, Forman D: Global cancer statistics. CA Cancer J Clin 2011, 61:69-90.

3. Li J, Jiang R, Liu WS, Liu Q, Xu M, Feng QS, Chen LZ, Bei JX, Chen $M Y$, Zeng $Y X$ : A large cohort study reveals the association of elevated peripheral blood lymphocyte-to-monocyte ratio with favorable prognosis in nasopharyngeal carcinoma. PLOS One 2013, 8(12):e83069.

4. He JR, Shen GP, Ren ZF, Qin H, Cui C, Zhang $Y$, Zeng $Y X$, Jia WH: Pretreatment levels of peripheral neutrophils and lymphocytes as independent prognostic factors in patients with nasopharyngeal carcinoma. Head Neck 2012, 34(12):1769-1776.
5. Tsai YD, Wang CP, Chen CY, Lin LW, Hwang TZ, Lu LF, Hsu HF, Chung FM, Lee YJ, Houng JY: Pretreatment circulating monocyte count associated with poor prognosis in patients with oral cavity cancer. Head Neck 2014 36(7):947-953.

6. Lu CC, Chang KW, Chou FC, Cheng CY, Liu CJ: Association of pretreatment thrombocytosis with disease progression and survival in oral squamous cell carcinoma. Oral Oncol 2007, 43:283-288.

7. Sierko E, Wojtukiewicz MZ: Platelets and angiogenesis in malignancy. Semin Thromb Hemost 2004, 30:95-108.

8. Ikeda M, Furukawa H, Imamura H, Shimizu J, Ishida H, Masutani S, Tatsuta M, Satomi T: Poor prognosis associated with thrombocytosis in patients with gastric cancer. Ann Surg Oncol 2002, 9(3):287-291.

9. Gucer F, Moser F, Tamussino K, Reich O, Haas J, Arikan G, Petru E, Winter R: Thrombocytosis as a prognostic factor in endometrial carcinoma. Gynecol Oncol 1998, 70(2):210-214.

10. Taucher S, Salat A, Gnant M, Kwasny W, Mlineritsch B, Menzel RC, Schmid M, Smola MG, Stierer M, Tausch C, Galid A, Steger G, Jakesz R, Austrian Breast and Colorectal Cancer Study Group: Impact of pretreatment thrombocytosis on survival in primary breast cancer. Thromb Haemost 2003, 89(6):1098-1106.

11. Kandemir EG, Mayadagli A, Karagoz B, Bilgi O, Turken O, Yaylaci M: Prognostic significance of thrombocytosis in node-negative colon cancer. J Int Med Res 2005, 33:228-235.

12. Yu D, Liu B, Zhang L, Du K: Platelet count predicts prognosis in operable non-small cell lung cancer. Exp Ther Med 2013, 5:1351-1354

13. Chen MH, Chang PM, Chen PM, Tzeng CH, Chu PY, Chang SY, Yang MH: Prognostic significance of a pretreatment hematologic profile in patients with head and neck cancer. J Cancer Res Clin Oncol 2009, 135(12):1783-1790.

14. Jurk K, Kehrel BE: Platelets: physiology and biochemistry. Semin Thromb Hemost 2005, 31:381-392.

15. de Gaetano G: Historical overview of the role of platelets in hemostasis and thrombosis. Haematologica 2001, 86:349-356.

16. Wakefield LM, Smith DM, Flanders KC, Sporn MB: Latent transforming growth factor-beta from human platelets. A high molecular weight complex containing precursor sequences. J Biol Chem 1988 263:7646-7654.

17. Banks RE, Forbes MA, Kinsey SE, Stanley A, Ingham E, Walters C, Selby PJ: Release of the angiogenic cytokine vascular endothelial growth factor (VEGF) from platelets: significance for VEGF measurements and cancer biology. Br J Cancer 1998, 77(6):956-964.

18. Pilatova K, Greplova K, Demlova R, Bencsikova B, Klement GL, Zdrazilova-Dubska L: Role of platelet chemokines, PF-4 and CTAP-III, in cancer biology. J Hematol Oncol 2013, 6:42.

19. Senzel L, Gnatenko DV, Bahou WF: The platelet proteome. Curr Opin Hematol 2009, 16:329-333.

20. Guo F, Parker Kerrigan BC, Yang D, Hu L, Shmulevich I, Sood AK, Xue F, Zhang W: Post-transcriptional regulatory network of epithelial-tomesenchymal and mesenchymal-to-epithelial transitions. J Hematol Oncol 2014, 7:19.

21. Saito Y, Haendeler J, Hojo Y, Yamamoto K, Berk BC: Receptor heterodimerization: essential mechanism for platelet-derived growth factor-induced epidermal growth factor receptor transactivation. Mol Cell Biol 2001, 21:6387-6394.

22. Labelle M, Begum S, Hynes RO: Direct signaling between platelets and cancer cells induces an epithelial-mesenchymal-like transition and promotes metastasis. Cancer Cell 2011, 20:576-590

23. Kaser A, Brandacher G, Steurer W, Kaser S, Offner FA, Zoller H, Theurl I, Widder W, Molnar C, Ludwiczek O, Atkins MB, Mier JW, Tilg H: Interleukin-6 stimulates thrombopoiesis through thrombopoietin: role in inflammatory thrombocytosis. Blood 2001, 98(9):2720-2725.

24. Suzuki A, Takahashi T, Nakamura K, Tsuyuoka R, Okuno Y, Enomoto $T$, Fukumoto $M$, Imura $H$ : Thrombocytosis in patients with tumors producing colony-stimulating factor. Blood 1992 80(8):2052-2059.

25. Algra AM, Rothwell PM: Effects of regular aspirin on long-term cancer incidence and metastasis: a systematic comparison of evidence from observational studies versus randomised trials. Lancet Oncol 2012, 13:518-527. 
26. Rothwell PM, Wilson M, Elwin CE, Norrving B, Algra A, Warlow CP, Meade TW: Long-term effect of aspirin on colorectal cancer incidence and mortality: 20-year follow-up of five randomised trials. Lancet 2010, 376(9754):1741-1750.

27. Ji X, Hou M: Novel agents for anti-platelet therapy. J Hematol Oncol 2011, 4:44.

doi:10.1186/s13045-014-0065-5

Cite this article as: Rachidi et al:: Lower circulating platelet counts and antiplatelet therapy independently predict better outcomes in patients with head and neck squamous cell carcinoma. Journal of Hematology \& Oncology 2014 7:65.

\section{Submit your next manuscript to BioMed Central and take full advantage of:}

- Convenient online submission

- Thorough peer review

- No space constraints or color figure charges

- Immediate publication on acceptance

- Inclusion in PubMed, CAS, Scopus and Google Scholar

- Research which is freely available for redistribution 